\title{
Nanoscale electrical conductivity of the purple membrane monolayer
}

\author{
I. Casuso, ${ }^{1}$ L. Fumagalli, ${ }^{1}$ J. Samitier, ${ }^{1}$ E. Padrós, ${ }^{2}$ L. Reggiani, ${ }^{3}$ V. Akimov, ${ }^{3}$ and G. Gomila ${ }^{1}$ \\ ${ }^{1}$ Deptartment Electrònica, Universitat de Barcelona and Laboratori de Nanobioenginyeria-IBEC, Parc Científic de Barcelona, \\ Barcelona, Spain \\ ${ }^{2}$ Unitat de Biofísica, Department de Bioquímica i de Biologia Molecular, Facultat de Medicina i Centre d'Estudis en Biofísica, \\ Universitat Autònoma de Barcelona, Barcelona, Spain \\ ${ }^{3}$ CNR-INFM National Nanotechnology Laboratory, Dipartimento di Ingegneria dell'Innovazione, Università di Lecce, Lecce, Italy
}

(Received 29 December 2006; revised manuscript received 27 September 2007; published 31 October 2007)

\begin{abstract}
Nanoscale electron transport through the purple membrane monolayer, a two-dimensional crystal lattice of the transmembrane protein bacteriorhodopsin, is studied by conductive atomic force microscopy. We demonstrate that the purple membrane exhibits nonresonant tunneling transport, with two characteristic tunneling regimes depending on the applied voltage (direct and Fowler-Nordheim). Our results show that the purple membrane can carry significant current density at the nanometer scale, several orders of magnitude larger than previously estimated by macroscale measurements.
\end{abstract}

DOI: 10.1103/PhysRevE.76.041919

PACS number(s): 87.80.-y, 73.40.Rw, 85.65.+h

The purple membrane (PM) is a two-dimensional crystal lattice naturally present in the cell membrane of Halobacterium salinarum. It is composed of a lipid bilayer and a single-protein species, the Bacteriorhodopsin (bR), in a lipidto-protein ratio of $10(\mathrm{~mol} / \mathrm{mol})$. Bacteriorhodopsin acts as a light-driven proton pump, converting solar energy into an electrochemical proton gradient across the cell membrane $[1,2]$. Its functional stability under different environmental conditions combined with easy and large production has made bR a model protein for studies of charge transport on cell membranes, as well as an excellent candidate for bioelectronic applications [3,4].

Despite its enormous interest, only a few studies regarding the electron transport measurements of a single PM layer have been reported so far, leading to an incomplete and controversial picture [5-7]. The main obstacle encountered in measuring the electrical conductivity of the PM monolayer $(\sim 5 \mathrm{~nm}$ thick) resides on providing reliable electrical contact at the electrode-membrane interface. Measured currents can dramatically differ by orders of magnitude from measurement to measurement on supposedly identical conditions, being extremely sensitive to the electrode-membrane distance as well as the applied load on the membrane. To date, two methods have been reported: (i) measuring the current of a monolayer confined between two submillimeter-sized electrodes [5] and (ii) probing the nanoscale conductivity of the monolayer using a scanning tunneling microscope (STM) $[6,7]$. The millimeter-sized electrode configuration demands a flat and hole-free monolayer covering the entire electrode surface, which is, however, difficult to fabricate. Furthermore, averaging of biological information on a macroscale level is inherent to this method. To study electron conduction at the molecular level, scanning probe techniques are by far the most appropriate approach. STM, however, has an intrinsic limit in the tunneling current feedback for insulating samples which impedes the control and quantification of the probe-membrane distance and forces applied on the biomolecules.In this article we use conductive atomic force microscopy (C-AFM) as an extremely controlled method to provide a comprehensive and unambiguous model of electron con- duction in the PM monolayer. Conductive AFM has demonstrated to be well suited to studying the electrical properties of biomolecules [8-10], offering significant advantages over the aforementioned techniques. The technique is based on a flexible conductive cantilever with a sharp tip connected to a current detector. The current flowing through the tip in contact with the sample is measured under force control. Optical detection of the cantilever deflection together with piezoelectric displacement of the tip allows sub-nm accuracy in vertical tip positioning and sub-nN control of the force applied on the monolayer. Furthermore, images of topography and electrical conduction at the nanoscale are simultaneously obtained.

For the experiments reported here, PMs containing wildtype $\mathrm{bR}$ are isolated from the bacterial cell membrane to a final concentration of $10^{-7} \mathrm{M}$ [11], deposited on a gold film evaporated on a mica substrate, dried under $\mathrm{N}_{2}$ flow, and then placed under the AFM chamber (Nanotec Electronica S.L). Measurements are performed under dry $\mathrm{N}_{2}$ atmosphere, to avoid water deposition on the PM patches [12]. Importantly, under dry conditions the integrity of the PM structure is preserved. Furthermore, the membrane shows functional photoelectric activity which in fact is used in most of the bioelectronic applications [2-4]. Conductive diamond-coated tips are employed (Nanosensors CDT) for superior wear resistance and long-term electrical conductivity. A custommade current transimpedance amplifier is used to perform dc current measurements in a wide dynamic range, from $\sim 0.3 \mathrm{pA}$ up to $10 \mathrm{nA}$. All current data presented here are raw data; only the constant amplifier offset has been subtracted.

Two representative conductive images of PM monolayer patches as obtained with C-AFM are given in Figs. 1(a) and 1(c). The images have been acquired at low applied bias $(100 \mathrm{mV})$ simultaneously with the topography [Figs. 1(b) and $1(\mathrm{~d})]$ by using a $0.2-\mathrm{N} / \mathrm{m}$ conductive tip (CDT-CONTR) and a maximum applied force of $3 \mathrm{nN}$. We have operated the AFM in jumping mode [13], achieving stable electrical contact during which the current is measured while avoiding shear forces during the lateral displacement. This allows con- 


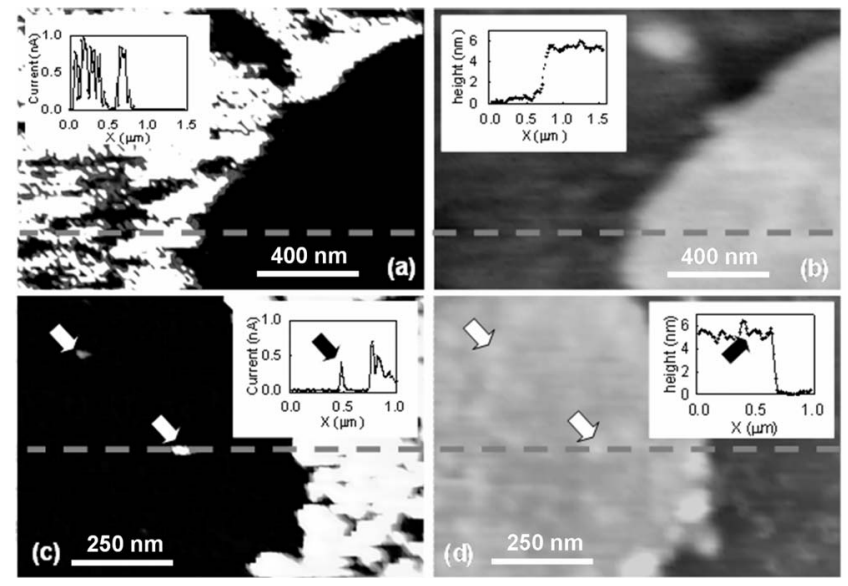

FIG. 1. Simultaneous conductive (a), (c) and topographic (b), (d) images of two PM monolayer patches on a gold substrate obtained in jumping mode at low applied bias $(100 \mathrm{mV})$. Note the strong contrast in conduction [(a) and (c)] between the membrane patch (black area) and the gold substrate (bright areas). In a few percent of the cases conductive defects not related to topographic features (arrows) are observed on the membrane patches [(c) and (d)]. The insets show the current and topography profiles for both membranes. PM thickness $\sim 5.5 \mathrm{~nm}$.

tinuous scan of a PM patch for hours without observable sample or image modification [14]. A trade-off between applied voltage and cantilever stiffness has been necessary to avoid sample damaging. In imaging mode a soft cantilever is necessary but this implies the use of low applied voltages to avoid excessive electrostatic bending of the cantilever [15]. The observed topography of the membrane shows the expected $\sim 5 \mathrm{~nm}$ thickness of the PM monolayer, as seen in the topography inset. On the other hand, the conductive image shows an insulating nature of the PM monolayer. On the vast majority of the PM patches analyzed the electron current levels are lower than the setup resolution-i.e., 0.3 pA. Only in approximately $5 \%$ of the PM monolayers have isolated conductive pinholes, not related to significant topographic features, been identified, Fig. 1(c). Although the origin of these conductive pinholes needs further investigation, its existence validates the measuring technique.

To investigate the conduction mechanism, current-voltage $I(V)$ characteristics at a fixed location of the PM monolayer have been measured. Previous C-AFM measurements on proteins used 2-N/m cantilevers and combined the dynamic mode for the positioning of the AFM tip in the $X Y$ plane, with a force feedback approach for the positioning the AFM tip on the $Z$ axis on top of the protein, and next performed the $I(V)$ characteristic. This strategy avoided damaging the sample, but at the expense of limiting the maximum applied bias to $1 \mathrm{~V}$ to avoid that the electrostatic force acting on the probe damaged the proteins during the $I(V)$ characteristic acquisition [8-10]. In our case, we have used stiff cantilevers of $40 \mathrm{~N} / \mathrm{m}$ instead; this has allowed us to extend the bias range from $1 \mathrm{~V}$ up to $9 \mathrm{~V}$. This has been possible because stiffer cantilevers absorb more electrostatic force and reduce the force transmitted to the sample during the $I(V)$ acquisition. The dynamic mode has also been used to position the
AFM tip in the $X Y$ plane with the stiff cantilevers and without damaging the PM patches. But the positioning of the tip in the $Z$ axis requires new strategies, since the low sensitivity to the applied force of the stiff cantilevers results in excessive forces on the PM patches during the force feedback approach, which results in damages to the samples. One possibility is the use of an open feedback stepwise approach where an $I(V)$ characteristic is performed at each step; this strategy, previously only used with $2-\mathrm{N} / \mathrm{m}$ cantilevers [16], works nicely with stiff cantilevers because the electrostatic cantilever bending at each step is greatly reduced and thus enables, step by step, a set of $Z$ positions covering all possible tip locations (that is, tip in the air, tip just on top of the sample, and the progressive indentation of the tip in the sample) to be electrically and individually characterized, with the advantage that at each step the $Z$ position can be directly related to the distance $L$ between the tip and the gold substrate through monitoring of the cantilever deflection and the piezovertical displacement. For our measurement, we used a step size of $0.1 \mathrm{~nm}$ and diamond-coated CDT-NCHR probes with nominal radius $100-200 \mathrm{~nm}$.

A three-dimensional (3D) linear representation of $I(V)$ curves measured in one full indentation process is given in Fig. 2(a). The measured current shows an exponential behavior as a function of the applied bias up to saturation of the current amplifier (10 nA) and centered at $0 \mathrm{~V}$. For increasing indentation depth the applied force grows linearly up to $40 \mathrm{nN}$ and the measured current progressively increases until the tip reaches the gold substrate. Figures 2(b) and 2(c) show $I(V)$ characteristics observed at different indentation depths selected from one 3D image and displayed in log-linear and Fowler-Nordheim representations, respectively. The solid lines are theoretical fittings of the experimental data, as discussed below. Figures 2(b) and 2(c) clearly show the existence of two different transport regimes and the transition between them depending on the applied bias.

These two electrical regimes are distinctive for nonresonant tunnel transport of thin insulating films. Electron tunneling through the energy barrier of an insulator in a metalinsulator-metal (MIM) system has been described by Simmons [17] and can be expressed by the $I(V)$ tunneling equation

$$
I=\frac{q A}{2 \pi h \bar{d}}\left[\bar{\phi} e^{-\left(4 \pi \sqrt{\left.2 m^{*} / h\right)} \bar{d} \sqrt{\bar{\phi}}\right.}-(\bar{\phi}+q V) e^{-\left(4 \pi \sqrt{2 m^{*}} / h\right) \bar{d} \sqrt{\bar{\phi}+q V}}\right]
$$

where $A$ is an effective electrical contact area, $q$ the electron charge, $m^{*}$ the effective electron mass, $V$ the applied voltage bias, and $h$ Planck's constant. Other important tunneling parameters are $\bar{d}$, the effective tunnel distance, and $\bar{\phi}$, the mean barrier height, which depend both on the metal-insulator contact barrier height $\phi_{c}$, the interelectrode distance $L$, and the applied bias. In the simplest case of a symmetric MIM system, assuming a linear voltage drop across the insulating barrier and neglecting space charge and image charge effects [17], it can be shown that 

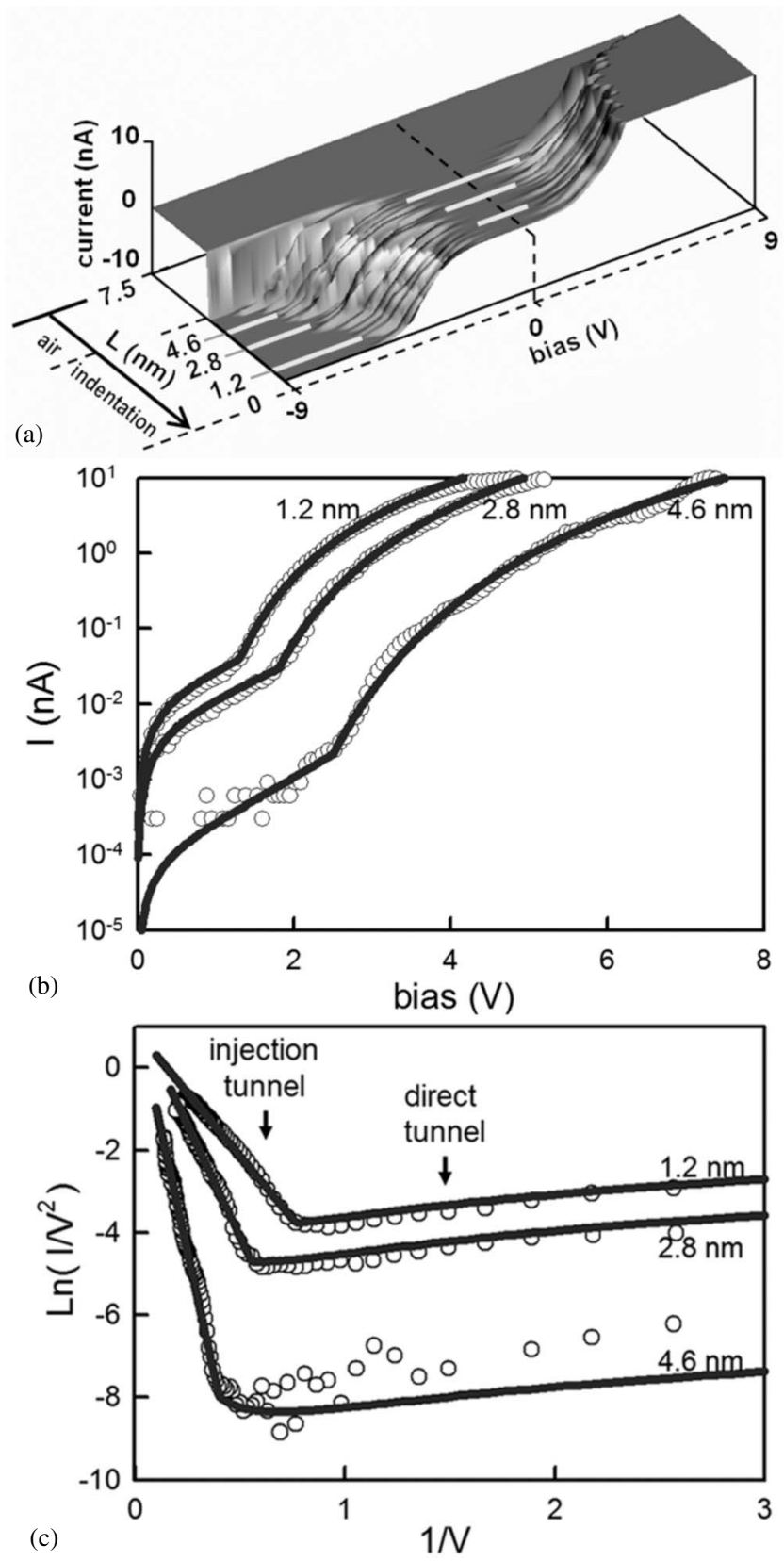

FIG. 2. (a) Three-dimensional representation of experimental $I(V)$ characteristics acquired at different interelectrode distances $L$ during indentation of the PM monolayer $(L \sim 5 \mathrm{~nm}$ corresponds to no indentation and $L=0$ to the tip in contact with the gold). (b) Log-linear and (c) Fowler-Nordheim representations of measured $I(V)$ characteristics (circles) selected from the 3D plot and acquired at different interelectrode distances $(4.6 \mathrm{~nm}, 2.8 \mathrm{~nm}$, and $1.2 \mathrm{~nm})$. Theoretical fitting curves are also given (solid lines). The data clearly show two conduction regimes depending on the applied bias, consistent with direct and injection (Fowler-Nordheim) tunneling.

$$
\bar{d}=\left\{\begin{array}{cc}
L, & q V<\phi_{c}, \\
\frac{\phi_{c}}{q V} L, & q V>\phi_{c},
\end{array} \quad \bar{\phi}=\left\{\begin{array}{cc}
\phi_{c}-\frac{q V}{2}, & q V<\phi_{c}, \\
\frac{\phi_{c}}{2}, & q V>\phi_{c} .
\end{array}\right.\right.
$$
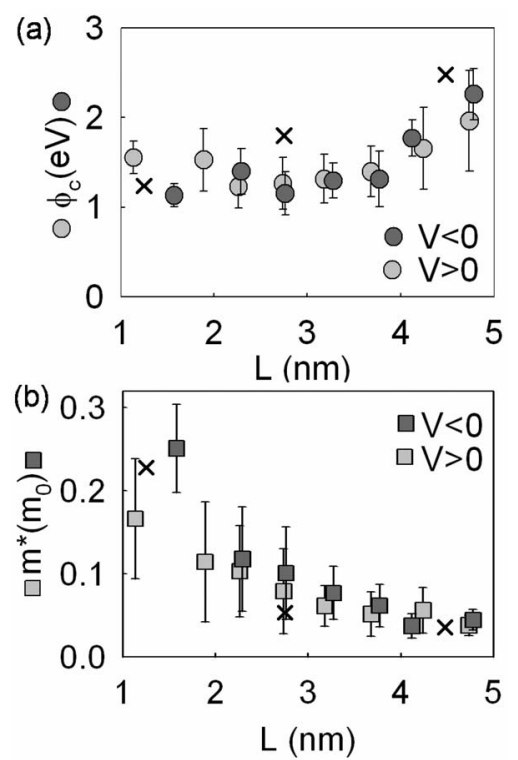

FIG. 3. Contact barrier height (a) and effective electron mass (b) as a function of the interelectrode distance $L$ for the electrons injected from substrate $(V<0)$ and from the tip $(V>0)$. The values are obtained from data fitting to Eq. (1) and correspond to the average of three independent acquisitions on different membrane patches. Bars represent the standard deviation. The precise values corresponding to the current-voltage curves in Fig. 1(b) are highlighted as crosses.

Therefore, Eq. (1) can be approximated as two transport regimes, depending on the applied bias as compared to the barrier height. When the bias is lower than the barrier height $\left(q V<\phi_{c}\right)$, electrons directly tunnel from metal to metal (direct tunnel regime). When the bias exceeds the barrier height $\left(q V>\phi_{c}\right)$, electrons are injected first into the insulator and then to the second electrode (injection tunnel or FowlerNordheim regime).

Excellent agreement of the experimental results with these two tunneling regimes for the entire applied bias range is obtained by fitting individual $I(V)$ observations to Eq. (1). To fit the data, we first estimate the contact barrier height $\phi_{c}$ from the transition voltage from the direct to injection regime. Then, we obtain the effective electron mass $m^{*}$ from the slope of the injection regime in the Fowler-Nordheim representation [Fig. 2(b)] [17], which can be expressed as

$$
s=-\frac{8 \pi \sqrt{2 m^{*}}}{h q}\left(\frac{\phi_{c}}{2}\right)^{3 / 2} L .
$$

Finally, the remaining parameter $A$, the effective contact area, is tuned until the theoretical curve matches exactly the observed $I(V)$. Figures 2(a) and 2(b) show that the model perfectly fits the data in the injection regime and often also matches the observed direct regime.

Figures 3(a) and 3(b) give the average of the values extracted for $\phi_{c}$ and $m^{*}$ as a function of the interelectrode distance $L$ and voltage polarity. Data are obtained from three independent acquisitions on different membrane patches. The observed barrier height $\phi_{c}$ is $2.2 \pm 0.6 \mathrm{eV}$ at the very begin- 
ning of PM indentation $(L=5 \mathrm{~nm})$ and decreases until $\sim 1.5 \pm 0.2 \mathrm{eV}$ at $L=3 \mathrm{~nm}$ at a rate of $0.6 \pm 0.2 \mathrm{eV} / \mathrm{nm}$ where it stabilizes. On the other hand, at the first tip-membrane contact, the observed electron mass $m^{*}$ is $(0.04 \pm 0.02) m_{0}$, increasing with the interelectrode distance at a rate $(2.7 \pm 2.3) \times 10^{-2} m_{0} / \mathrm{nm}$ until $L \sim 2.5 \mathrm{~nm}$, where it sharply increases up to $\sim(0.2 \pm 0.1) m_{0}$. Only a slight asymmetry in the effective mass depending on the bias polarity can be noticed. Higher values of $m^{*}$ are found when electrons are injected from the substrate towards the tip $(V<0)$, which we attribute to the unequal fields at the electrode-membrane interface $[8,9]$. Finally, the effective contact area $A$ lowers from $\sim 0.1 \mathrm{~nm}^{2}$ at the beginning of the indentation to $\sim 0.01 \mathrm{~nm}^{2}$ for $L<2 \mathrm{~nm}$.

Thanks to ability of observing the transition from direct to injection tunneling in biolayers, previously only observed in a self-assembled monolayer (SAM) system [18], we have been able to uncouple the effective mass and height of the energy barrier and thus complete previous discussions exclusively in terms of barrier height $[8,9]$. The results demonstrate that the mechanism of electron conduction through the PM monolayer is nonresonant tunneling for the entire applied bias range, in agreement with established tunneling $L$-related tendencies observed in oxide systems for $\phi_{c}$ [19] and $m^{*}[20]$. Furthermore, the results are consistent with the expected dependence of $\phi_{c}$ on the protein atomic packing [8].

We also remark that from our experimental data on dried PM there is no evidence of electron transport processes mediated by inner conduction pathways in the PM monolayer at $40 \%$ relative humidity $(\mathrm{RH})[5]$, which would indicate that the water content in proteins has an important role in the determination of the electron conduction mechanism in proteins, even if the PM is functional both dried and at $40 \% \mathrm{RH}$. We note that the electron effective mass in PM is one order of magnitude smaller than those found in SAMs and very thin oxide films [21,22] (to our knowledge no such data for biological samples have been reported). This value of $m^{*}$ explains the rather high current levels measured through the PM monolayer. The measured effective contact area parameter is found to be small, but in agreement with characteristic field emission areas [23], and the observed area variation agrees with extended FN theories for hemiellipsoidal electrode geometries [24]. From the observed contact area of the monolayer $\left(0.1 \mathrm{~nm}^{2}\right)$, we can estimate the current flow carried by an individual protein unit forming the purple mem- brane, the Bacteriorhodopsin trimer. Considering a trimer area of $\sim 25 \mathrm{~nm}^{2}$ and a current density of $\sim 2 \mathrm{pA} / \mathrm{nm}^{2}$ measured at $1 \mathrm{~V}$ [see Fig. 2(b)] we obtain a current flow of $50 \mathrm{pA}$ per trimer. We note that this is a significant current level, which is consistent with the observations of other proteins $[8-10]$. In contrast, the current flow reported through a single bR trimer using a submillimetre electrode configuration is several orders of magnitude smaller $\left(\sim 3 \times 10^{-19} \mathrm{~A}\right)$ [5]. The reason for this discrepancy might be due to a nonhomogenous current flow for the entire monolayer, due to larger interelectrode distance in some spots or to the quality of the membrane-electrode system. In our experiment we measure at the nanoscale, thus overcoming the problems of interelectrode distance variations and other potential sources of macroscale artifacts.

This article is framed in the context of previous experiments on electron transport mechanisms through protein systems; these experiments have reported a variety other transport mechanisms for other protein systems: Pole-Frenkel, semiconduction, photovoltaic effect, and hopping [25-27]. Direct nonresonant tunneling has been reported for two other protein systems $[8,9]$ but this article represents the most solid evidence of direct nonresonant tunneling in a protein to date and the only one for a protein system which does not contain metal atoms $[8,9]$.

In conclusion, we have studied the electrical transport mechanism of the purple membrane monolayer at the nanometer scale using a conductive AFM approach that guarantees accurate control of the probe-membrane distance, a parameter not controlled in previous works $[8-10,18]$. Nonresonant tunneling has been unambiguously demonstrated to be the electrical conduction mechanism through the PM monolayer in dried conditions. The same method can be also applied to study the electrical transport of other soft materials for biomolecular electronic applications.

Financial support by Grants Nos. BMC2003-04941 and NAN2004-09415-C05-01 of the Spanish Dirección General de Investigación (MCYT) and by the SPOT-NOSED European Project (No. IST-2001-388899) is gratefully acknowledged. We are grateful to Professor J. Gomez (Universitat Autómoma Madrid), Professor R. Reifenberger (Purdue University), Professor M. Sampietro and Dr. G. Ferrari (Politecnico di Milano), and Professor M. García-Parajó (IBEC, Barcelona) for invaluable assistance.
[1] D. Oesterhelt and W. Stoeckenius, Proc. Natl. Acad. Sci. U.S.A. 70, 2853 (1973).

[2] G. Varo and L. Keszthelyi, Biophys. J. 43, 47 (1983).

[3] J. Shin et al., Opt. Lett. 32, 500 (2007).

[4] J. Xu, P. Bhattacharya, and G. Varo, Biosens. Bioelectron. 19, 885 (2004).

[5] Y. Jin et al., Proc. Natl. Acad. Sci. U.S.A. 103, 8601 (2006).

[6] R. Guckenberger et al., J. Vac. Sci. Technol. B 9, 1227 (1991).

[7] R. García et al., Langmuir 11, 2109 (1995).
[8] J. Zhao et al., J. Am. Chem. Soc. 126, 5601 (2004).

[9] L. Andolfi, A. R. Bizzarri, and S. Cannistraro, Appl. Phys. Lett. 89, 183125 (2006).

[10] A. Stamouli et al., FEBS Lett. 560, 109 (2004).

[11] D. Oesterhelt and W. Stoeckenius, Methods Enzymol. 31, 667 (1974).

[12] A. Gil et al., Appl. Phys. A: Mater. Sci. Process. 72, 137 (2001).

[13] F. Moreno-Herrero, J. Colchero, J. Gomez-Herrero, and A. M. 
Baro, Phys. Rev. E 69, 031915 (2004).

[14] See EPAPS Document No. E-PLEEE8-76-164710 for images demonstrating that the AFM study does not alter the topology of the membrane. For more information on EPAPS, see http:// www.aip.org/pubserves/epaps.html

[15] J. Toset et al., Nanotechnology 17, 1 (2006).

[16] C. Gomez-Navarro et al., Nanotechnology 13, 314 (2002).

[17] J. G. Simmons, J. Appl. Phys. 34, 1793 (1963).

[18] J. M. Beebe, B. S. Kim, J. W. Gadzuk, C. D. Frisbie, and J. E. Kushmerick, Phys. Rev. Lett. 97, 026801 (2006).

[19] G. Lewicki and J. Maserjian, J. Appl. Phys. 46, 3032 (1975)

[20] M. Stadele et al., J. Appl. Phys. 93, 2681 (2003).
[21] W. Y. Wang, T. Lee, and M. A. Reed, Rep. Prog. Phys. 68, 523 (2005).

[22] B. Brar, G. D. Wilk, and A. C. Seabaugh, Appl. Phys. Lett. 69, 2728 (1996).

[23] C. A. Spindt et al., J. Appl. Phys. 47, 5248 (1976).

[24] J. D. Zuber, K. L. Jensen, and T. E. Sullivan, J. Appl. Phys. 91, 9379 (2002).

[25] S. A. Campbell et al., J. Electroanal. Chem. 599, 313 (2007).

[26] D. Sarkar and T. N. Misra, J. Chem. Soc., Faraday Trans. 88, 53 (1992).

[27] P. B. Lukins, Chem. Phys. Lett. 321, 13 (2000). 